\title{
TT Arietis: Photometric variability from 1985 to 1994
}

\author{
Z. Kraicheva, V. Stanishev, L. Iliev, A. Antov, and V. Genkov \\ Institute of Astronomy, Bulgarian Academy of Sciences, 72 Tsarigradsko Shosse Blvd., BG-1784 Sofia, Bulgaria
}

Received February 21; accepted May 31, 1996

\begin{abstract}
Results of photoelectric observations of TT Ari during the 1985-1994 interval are presented. Observations were carried out at the Rozhen and Belogradchik observatories as part of our programme for the study of anti-dwarf novae. The long-term light curve in the standard $U B V$ system is constructed and peculiarities of the long-term behaviour of the star in this time interval are discussed. 21 moments of maxima and 14 moments of minima are added to the list of known maxima and minima of 3.2-hour modulations. The period of 3.2hour modulations during the $1987 / 1988,1989 / 1990$ and $1990 / 1991$ seasons is determined. In our 29 observing runs we searched for 20-min quasi-periodic variations, which were detected in 25 of them. Our results show that in 1989 , the 20-min modulation mean period reached a local maximum of $19.45 \mathrm{~min}$ and declined afterwards. The mean brightness variability analysis during the aforementioned seasons confirmed the reliability of the 3.8 period suspected by Semeniuk et al. (1987).
\end{abstract}

Key words: accretion, accretion disks - stars: TT Ari - stars: cataclysmic variables - X-ray: stars

\section{Introduction}

The cataclysmic binary TT Arietis has been classified over the last years as an anti-dwarf nova (or VY Scl system, Ritter 1990). Some of the problems encountered in the investigation of the star, as is the case with most of the cataclysmic variables, are associated with finding reliable orbital parameters which are difficult to determine because of the complicated structure of the circumstellar matter, resulting in a great diversity of observational manifestations. Thus the questions about the TT Ari long-term behaviour of the 20-min, 3.2-hour and 4-day photometric periods, are still open in spite of the numerous contributions to the investigation of the brightness variability of the star.

Send offprint requests to: Z. Kraicheva
Cowley et al. (1975) and Thorstensen et al. (1985) determined the spectroscopic orbital period of TT Ari to be 0d13755. Smak \& Stepien (1969) found regular brightness variations with a period 0.1329 in the high state and reported quasi-periodic variations with a time scale from 14 to $20 \mathrm{~min}$.

The star became an object of intensive investigation after 1980, when its brightness decreased to $V \sim 14^{\mathrm{m}}$ and the transition to its first registered low state $\left(V \sim 16^{\mathrm{m}} 5\right)$ begun. Until then, only irregular variations between $V \sim 10.5$ and $V \sim 13 \mathrm{~m}^{\mathrm{m}} 0$ had been observed (Hudec et al. 1984). Udalski (1988) found an increase of the photometric 3.2 period by about $0.15 \%$ from 1985 to 1986. Observations of Volpi et al. (1988), carried out in October 1986, are in favour of the period of 0.13298 for these modulations but the authors could not completely exclude the period $P_{\mathrm{ph}}=0 \mathrm{~d} 13232$. Hollander \& van Paradijs (1992) established quasi-periodic brightness modulations with a period $15 \mathrm{~m} 3$ in their 1988 observing runs and confirmed the rapid period decrease, found by Semeniuk et al. (1987). They discussed the known accretion disc instability models and concluded that none of them could entirely explain this trend of the oscillations in TT Ari.

In this paper we present our photoelectric observations of TT Ari that provide information on the light-curve behaviour in the last ten years.

\section{Observations}

The data consist of 29 observing runs in the $U$ and $B$ photometric bands obtained from 1985 to 1994 . The duration of the runs varied from 55 min to 5 hours. Furthermore, there are 6 additional observational series in the standard $U B V$ system, 5 of them longer than 3 hours. All observations were carried out with the one-channel photometers attached to the $60-\mathrm{cm}$ Carl Zeiss telescopes of the Rozhen and Belogradchik Observatories of the BAS. The observations were processed with the reduction software described in Kirov et al. (1991). The star "c" of the sequence of Götz (1985) was used as a comparison star. The magnitudes of this star were taken from Shafter et al. (1985). The integration time was $10 \mathrm{~s}$. 
Table 1. Observational series of TT Arietis

\begin{tabular}{|c|c|c|c|c|c|c|c|c|c|}
\hline$\overline{\mathrm{No}}$ & UT date & $\begin{array}{c}\text { Start time } \\
\text { [UT] }\end{array}$ & $\begin{array}{l}\text { Length } \\
\text { [hours] }\end{array}$ & Filter & No & UT date & $\begin{array}{c}\text { Start time } \\
{[\mathrm{UT}]}\end{array}$ & $\begin{array}{l}\text { Length } \\
\text { [hours] }\end{array}$ & Filter \\
\hline 1 & 1986 Nov. 02 & $21^{\mathrm{h}} 00^{\mathrm{m}}$ & $1^{\mathrm{h}} 00^{\mathrm{m}}$ & $U$ & 19 & 1990 Oct. 13 & $00^{\mathrm{h}} 30^{\mathrm{m}}$ & $1^{\mathrm{h}} 17^{\mathrm{m}}$ & $U$ \\
\hline 2 & 1987 Aug. 25 & $23^{\mathrm{h}} 30^{\mathrm{m}}$ & $2^{\mathrm{h}} 05^{\mathrm{m}}$ & $U$ & 20 & 1990 Oct. 13 & $23^{\mathrm{h}} 55^{\mathrm{m}}$ & $2^{\mathrm{h}} 05^{\mathrm{m}}$ & $U$ \\
\hline 3 & 1987 Aug. 31 & $23^{\mathrm{h}} 10^{\mathrm{m}}$ & $2^{\mathrm{h}} 05^{\mathrm{m}}$ & $U$ & 21 & 1990 Oct. 14 & $02^{\mathrm{h}} 40^{\mathrm{m}}$ & $1^{\mathrm{h}} 08^{\mathrm{m}}$ & $U$ \\
\hline 4 & 1987 Sep. 01 & $21^{\mathrm{h}} 55^{\mathrm{m}}$ & $2^{\mathrm{h}} 45^{\mathrm{m}}$ & $U$ & 22 & 1990 Oct. 15 & $22^{\mathrm{h}} 40^{\mathrm{m}}$ & $3^{\mathrm{h}} 40^{\mathrm{m}}$ & $U$ \\
\hline 5 & 1987 Dec. 07 & $20^{\mathrm{h}} 15^{\mathrm{m}}$ & $3^{\mathrm{h}} 40^{\mathrm{m}}$ & $U$ & 23 & 1991 Jan. 25 & $18^{\mathrm{h}} 05^{\mathrm{m}}$ & $2^{\mathrm{h}} 20^{\mathrm{m}}$ & $U$ \\
\hline 6 & 1987 Dec. 12 & $20^{\mathrm{h}} 35^{\mathrm{m}}$ & $1^{\mathrm{h}} 20^{\mathrm{m}}$ & $U$ & 24 & 1991 Jan. 26 & $17^{\mathrm{h}} 45^{\mathrm{m}}$ & $2^{\mathrm{h}} 35^{\mathrm{m}}$ & $U$ \\
\hline 7 & 1988 Jan. 18 & $17^{\mathrm{h}} 40^{\mathrm{m}}$ & $2^{\mathrm{h}} 20^{\mathrm{m}}$ & $U$ & 25 & 1992 Jan. 27 & $19^{\mathrm{h}} 50^{\mathrm{m}}$ & $0^{\mathrm{h}} 55^{\mathrm{m}}$ & $U$ \\
\hline 8 & 1988 Aug. 20 & $22^{\mathrm{h}} 50^{\mathrm{m}}$ & $2^{\mathrm{h}} 30^{\mathrm{m}}$ & $U$ & 26 & 1992 Dec. 18 & $18^{\mathrm{h}} 55^{\mathrm{m}}$ & $3^{\mathrm{h}} 35^{\mathrm{m}}$ & $U$ \\
\hline 9 & 1988 Sep. 12 & $22^{\mathrm{h}} 55^{\mathrm{m}}$ & $3^{\mathrm{h}} 20^{\mathrm{m}}$ & $B$ & 27 & 1992 Dec. 19 & $18^{\mathrm{h}} 00^{\mathrm{m}}$ & $2^{\mathrm{h}} 05^{\mathrm{m}}$ & $B$ \\
\hline 10 & 1988 Sep. 13 & $22^{\mathrm{h}} 40^{\mathrm{m}}$ & $2^{\mathrm{h}} 40^{\mathrm{m}}$ & $B$ & 28 & 1993 Oct. 13 & $22^{\mathrm{h}} 52^{\mathrm{m}}$ & $2^{\mathrm{h}} 42^{\mathrm{m}}$ & $U$ \\
\hline 11 & 1989 Aug. 01 & $23^{\mathrm{h}} 47^{\mathrm{m}}$ & $1^{\mathrm{h}} 30^{\mathrm{m}}$ & $U$ & 29 & 1994 Dec. 04 & $19^{\mathrm{h}} 37^{\mathrm{m}}$ & $2^{\mathrm{h}} 18^{\mathrm{m}}$ & $B$ \\
\hline 12 & 1989 Aug. 28 & $22^{\mathrm{h}} 10^{\mathrm{m}}$ & $3^{\mathrm{h}} 00^{\mathrm{m}}$ & $U$ & & & & & \\
\hline 13 & 1989 Oct. 24 & $20^{\mathrm{h}} 50^{\mathrm{m}}$ & $4^{\mathrm{h}} 00^{\mathrm{m}}$ & $U$ & 30 & 1985 Aug. 22 & $22^{\mathrm{h}} 50^{\mathrm{m}}$ & $3^{\mathrm{h}} 00^{\mathrm{m}}$ & $U B V$ \\
\hline 14 & 1989 Oct. 25 & $19^{\mathrm{h}} 50^{\mathrm{m}}$ & $5^{\mathrm{h}} 00^{\mathrm{m}}$ & $U$ & 31 & 1988 Oct. 09 & $23^{\mathrm{h}} 42^{\mathrm{m}}$ & $2^{\mathrm{h}} 30^{\mathrm{m}}$ & $U B V$ \\
\hline 15 & 1989 Oct. 28 & $20^{\mathrm{h}} 30^{\mathrm{m}}$ & $1^{\mathrm{h}} 20^{\mathrm{m}}$ & $B$ & 32 & 1989 Oct. 26 & $19^{\mathrm{h}} 18^{\mathrm{m}}$ & $5^{\mathrm{h}} 00^{\mathrm{m}}$ & $U B V$ \\
\hline 16 & 1989 Oct. 29 & $21^{\mathrm{h}} 00^{\mathrm{m}}$ & $3^{\mathrm{h}} 55^{\mathrm{m}}$ & $B$ & 33 & 1989 Oct. 27 & $19^{\mathrm{h}} 05^{\mathrm{m}}$ & $4^{\mathrm{h}} 00^{\mathrm{m}}$ & $U B V$ \\
\hline 17 & 1990 Jan. 24 & $18^{\mathrm{h}} 57^{\mathrm{m}}$ & $1^{\mathrm{h}} 20^{\mathrm{m}}$ & $U$ & 34 & 1993 Oct. 12 & $22^{\mathrm{h}} 12^{\mathrm{m}}$ & $3^{\mathrm{h}} 40^{\mathrm{m}}$ & $U B V$ \\
\hline 18 & 1990 Oct. 11 & $23^{\mathrm{h}} 04^{\mathrm{m}}$ & $2^{\mathrm{h}} 00^{\mathrm{m}}$ & $U$ & 35 & 1993 Oct. 27 & $20^{\mathrm{h}} 24^{\mathrm{m}}$ & $3^{\mathrm{h}} 20^{\mathrm{m}}$ & $U B V$ \\
\hline
\end{tabular}

The whole available observing data were used for the analysis of the photometric behavior of TT Ari. Details of the observing runs are given in Table 1. Apart from this series, there are more than 300 individual measurements in the standard $U B V$ system. Part of them have been already published by Kraicheva et al. (1987) and Kraicheva et al. (1989). The rest will be presented in a forthcoming publication. All published photometrical observations of TT Ari that contain useful information have been collected and also used in the analysis.

\section{Results and discussion}

\subsection{The long-term light curve of TT Arietis from 1985 to 1994}

To study the long-term photometric behavior of TT Ari, we used our $U B V$ measurements, obtained in the period 1985 - 1994. The $U B V$ light curve in this time interval is shown in Fig. 1. The interval JD $2446250-2447100$ covers the ascending branch top end of the long-term light curve after the star had left its 1979 - 1985 low state. Kraicheva et al. (1989) showed that the star reached its maximal brightness gradually: in a time interval of two years, the brightness increased by about 0.2 in the $B$ and $V$ bands and by $0 .{ }^{\mathrm{m}} 3$ in the $U$ band. Since 1987, TT Ari has been mainly in a high state and its brightness fluctuated about the mean values of almost $9{ }^{\mathrm{m}} 6$ in $U, 10^{\mathrm{m}} 6$ in $B$ and $10 \mathrm{~m} 8$ in $V$. The fluctuations around these mean values were considerable, and in two nights (JD 2448151 and 2448176 ) the brightness of TT Ari reached values typical for the intermediate state: 10.4 in $U, 11^{\mathrm{m}} 1$ in $B$ and $11^{\mathrm{m}} 2$

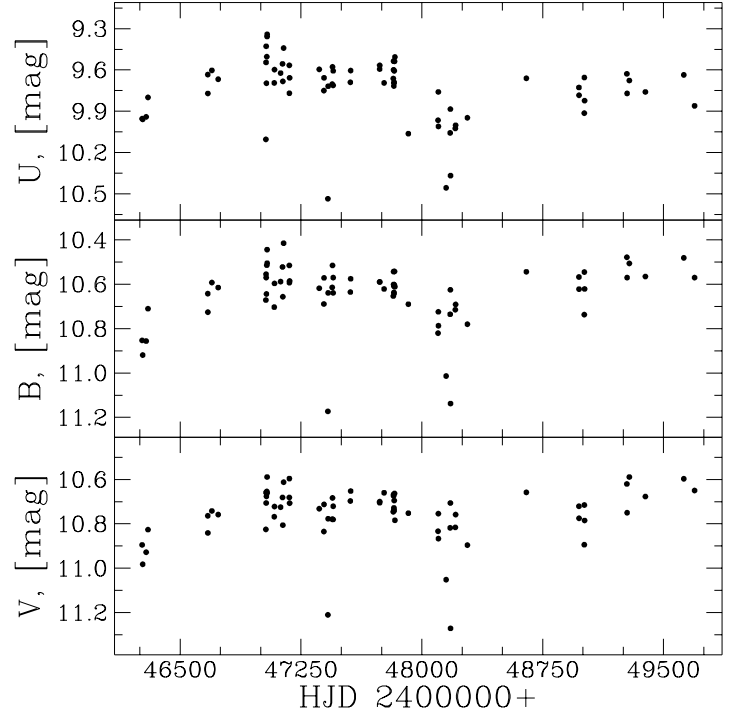

Fig. 1. TT Ari long-term light curve for the 1985-1994 time interval

in $V$. Unfortunately, the time interval when TT Ari was supposed to be in an intermediate state is not very well covered by our observations. There is a gap in the observations between JD 2448283 and 2448650 and it makes it impossible to describe how and when the star did return to its high state. It is interesting to note that the star returned to its previous high state mean brightness in the $B$ and $V$ bands, while in the $U$ band, TT Ari stayed at 
an average of 9.76 which is by $0 .{ }^{\mathrm{m}} 16$ fainter as compared with the previous high state value.

Our light curve continues the curves given in Hudec et al. (1984) and Wenzel et al. (1992). Their sum gives an exhaustive description of the photometric behavior of TT Ari from 1928 to the present time.

\subsection{The 20-min quasi-periodic variability}

We used two different methods to determine the 20min oscillations. They are the maximum entropy method (MEM) described, e.g., by Burg (1972) and Laccos (1971), and the period dispersion minimization method (PDM) described by Stellingwerf (1978).

By using these methods, we computed the MEM power spectra and $\theta$-statistics for the individual measurement series (from Nos. 1 to 29) listed in Table 1. To increase the accuracy in the determination of the 20-min oscillations periods, the influence of the 3-hour modulation was removed.

The peak corresponding to the 20-min oscillations is well seen in 21 nights both in the power spectrum and $\theta$-statistics. On four other nights it is seen either in the $\theta$-statistics or in the power spectrum. In most of the power spectra and $\theta$-statistics, this peak is accompanied by peaks that are comparable or even bigger, than that of the 20min oscillations. Their positions are highly variable and different in nights during a given observing season. We accepted the recurrence of the peak position as a criterion for the presence of quasi-periodic oscillations. The MEM power spectrum and $\theta$-statistics for the observing run No. 16, showing the longest period, are shown in Fig. 2.

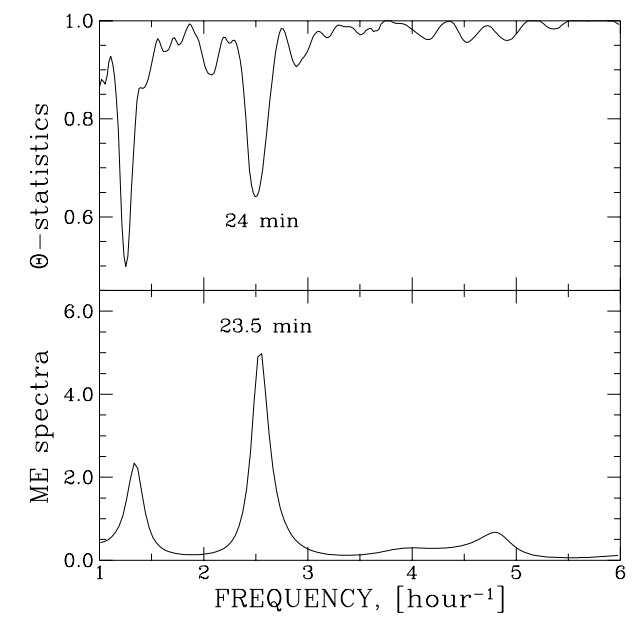

Fig. 2. Frequency analysis of observing run of Oct. 29, 1989

The values of the period obtained for the 20-min oscillations by the two methods are in good agreement. Using the PDM method we computed also the $\theta$-statistics for several nights in close succession of a given season. The results of the observations in the $U$ band are presented in Fig. 3. The mean amplitude of quasi-periodic brightness variations, obtained by a sinusoidal approximation, varied in the interval $0.024-0.035$ mag.

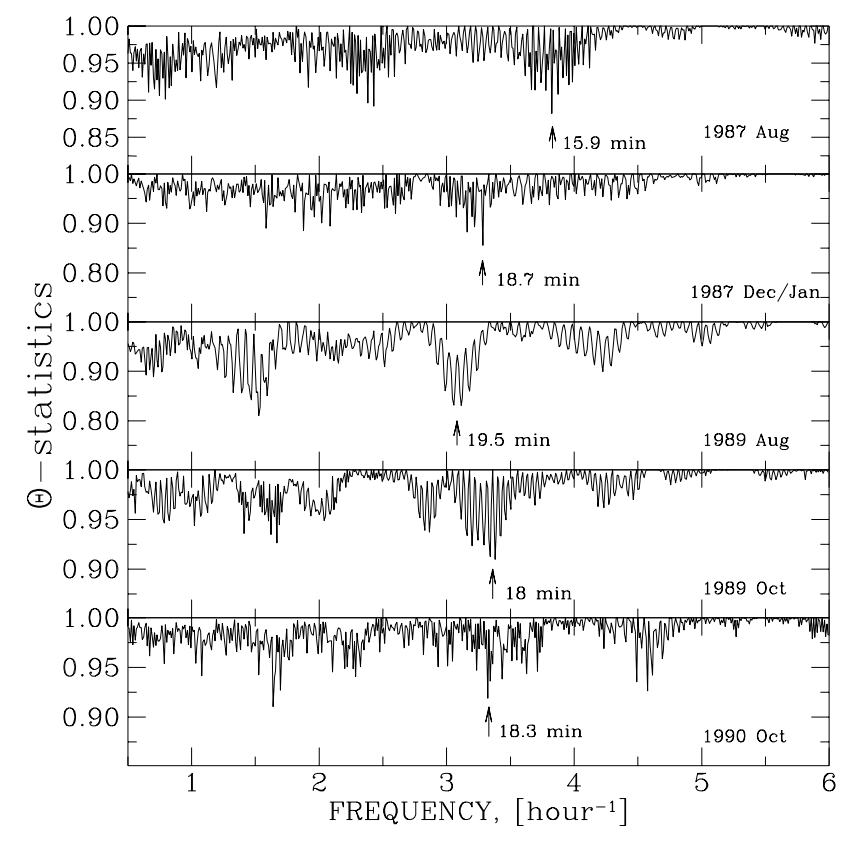

Fig. 3. The averaged seasonal behaviour of the TT Ari 20-min period

Semeniuk et al. (1987) found that the period of 20-min oscillations in TT Ari decreased from $27 \mathrm{~min}$ in 1961 to $17 \mathrm{~min}$ in 1985. Udalski (1988) found values for the $1987 / 88$ season in the $18-22$ min interval. We extended the curve of long-term changes of the 20-min oscillations given in Semeniuk et al. (1987) with our period values for the individual nights found by using the PDM and MEM methods. In our opinion, they give a more realistic picture of the behaviour of the 20-min period changes. The data are shown in Fig. 4. In the figure are also plotted the period values obtained by Volpi et al. (1988) and Hollander \& van Paradijs (1992), as well as the range in which the period varied according to Udalski (1988). Fortunately, we had observing series in the time interval close to the observing runs carried out by Volpi et al. (1988) and Hollander \& van Paradijs (1992). The coincidence with their results is excellent. In Figs. 3 and 4 it can be seen that the period of quasi-periodic oscillations did not decrease continuously. From 1987 to 1990 there was a noticeable increase of the mean period, but we have not enough observations for a detailed study of its long-term behaviour.

An interesting feature in the power spectra and $\theta$ statistics corresponds to a period of about $13 \mathrm{~min}$. We discovered it on 4 of the nights, where the 20-min oscillations were not clearly established. An inspection of the results for other observing nights shows that in 14 of the 
remaining observing nights the peak representing a 13-min periodicity can be either clearly seen or there are indications of it.

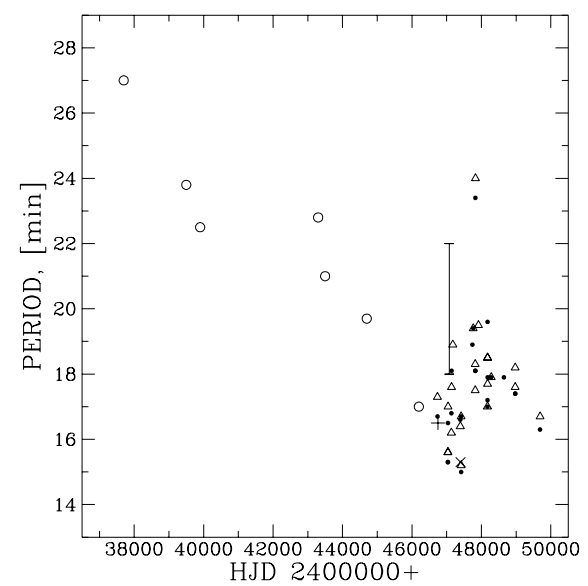

Fig. 4. The long-term behaviour of TT Ari 20-min period: - - our values found by MEM; $\triangle$ - our values found by PDM; ○ - the data of Semeniuk et al. (1987); + - the value of Volpi et al. (1988); $\times$ - the value of Hollander \& van Paradijs (1992); | - interval in which the period varied according to Udalski (1988)

\subsection{The three-hour photometric period}

In all observing runs of sufficient length 3-hour modulations are clearly seen. However, their amplitude and shape changed from night to night. The star also showed variations in its mean brightness. We applied the method of extrema for the 3-hour period determination, for observations in the seasons $1987 / 88,1989 / 90$ and 1990/91.

For TT Ari, as is the case with the other novalike stars, the determination of moments of extremum of the brightness was embarrassed by the strong flickering activity and the 20-min quasi-periodic oscillations. In some of the cases the flickering amplitude was bigger than the amplitude of the 3-hour modulation. Therefore we had to smooth the light curves in order to prepare them for a period analysis. For the purpose we used the method of moving average. The smoothing windows were equal to the characteristic time of 20-min quasi-periodic oscillations for the studied season, the step was $1 \mathrm{~min}$. The exact moments of extrema were determined by polynomial approximation.

Since the observations were carried out with the onechannel photometers, there were time gaps in the observing runs during the time of background and comparison star measurements. In order to perform the smoothing procedure, we filled the gaps by linearly interpolating the data. Our observing material, (Table 1), enabled us to determine 21 moments of maximum and 14 moments of minimum brightness, which can be found in Table 2 .
To determine the photometric period in the $1987 / 88$ season, we used the moments of maxima of brightness published by Udalski (1988) for the same interval plus moments determined by our series 3, 4, 5 and 7 (see Table 1). The cycle numbers $E$, corresponding to the four maxima calculated from observational runs, were determined by the photometric period found by Udalski (1988) $P_{\mathrm{ph}}=0$ d.132957. Moments of maximum covered relatively regularly the 165-day observation time interval. This gave the opportunity to determine accurately the cycle numbers. The following ephemeris were obtained by the least square method:

$M_{\max }[\mathrm{HJD}]=2447031.5863+0.132946 E$.

The (O-C) values obtained according to this ephemeris are shown in Fig. 5. Generally they are less than 0.11. The two exceptions, 0.15 and 0.17 , are probably due to the high flickering activity during the nights. The data of Udalski (1988) are also compatible with a period of 0.13265 . We examined periods of $0.1326,0.1327$ and 0.1328 . It was found that the values of $(\mathrm{O}-\mathrm{C})$ for the last three periods were much higher than for the first period. Therefore, in our further discussion we concentrated on the period of 0 d 132946.

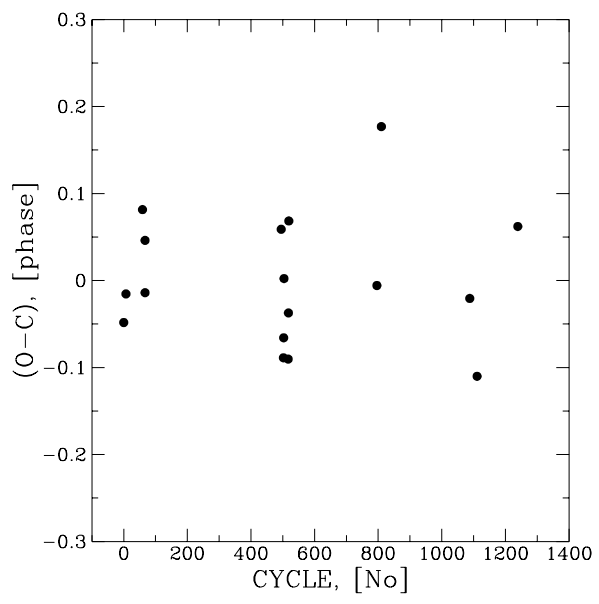

Fig. 5. (O-C) diagram for the $1987 / 88$ season computed by using Eq. (1)

Our observing material for the $1989 / 90$ season enabled us to determine five moments of maximum and five moments of minimum of the three-hour modulations. This was an interesting possibility to compare periods obtained from two extremum systems of the same observing data. All maxima were found from the observing series 13, 14 and 16. Four moments of minimum were obtained from the observing runs $12,13,14$ and 16 and one by the $U B V$ measurements of October 26, 1989 (Table 1). Four of them are within a 5-day time interval which gives a certain 
Table 2. Moments of extrema of 3.2 hour wave

\begin{tabular}{|c|c|c|c|c|c|c|c|}
\hline$\overline{\mathrm{No}}$ & UT date & $\begin{array}{c}\text { Moments of extrema } \\
\text { HJD } 2440000+\end{array}$ & Type & No & UT date & $\begin{array}{l}\text { Moments of extrema } \\
\text { HJD } 2440000+\end{array}$ & Type \\
\hline 1 & 1985 Aug. 22 & 6300.525 & $\max$ & 19 & 1993 Oct. 12 & 9273.514 & $\max$ \\
\hline 2 & 1987 Aug. 31 & 7039.441 & $\max$ & 20 & 1993 Oct. 27 & 9288.383 & $\max$ \\
\hline 3 & 1987 Sep. 01 & 7040.500 & $\max$ & 21 & 1994 Dec. 04 & 9691.353 & $\max$ \\
\hline 4 & 1987 Dec. 07 & 7137.411 & $\max$ & & & & \\
\hline 5 & 1988 Jan. 18 & 7179.276 & $\max$ & 22 & 1987 Aug. 25 & 7033.512 & $\min$ \\
\hline 6 & 1988 Sep. 12 & 7417.541 & $\max$ & 23 & 1988 Jan. 18 & 7179.314 & $\min$ \\
\hline 7 & 1988 Sep. 13 & 7418.487 & $\max$ & 24 & 1988 Aug. 20 & 7394.491 & $\min$ \\
\hline 8 & 1989 Oct. 24 & 7824.474 & $\max$ & 25 & 1988 Sep. 13 & 7418.532 & $\min$ \\
\hline 9 & 1989 Oct. 25 & 7825.384 & $\max$ & 26 & 1989 Aug. 28 & 7767.487 & $\min$ \\
\hline 10 & 1989 Oct. 25 & 7825.518 & $\max$ & 27 & 1989 Oct. 24 & 7824.421 & $\min$ \\
\hline 11 & 1989 Oct. 29 & 7829.406 & $\max$ & 28 & 1989 Oct. 25 & 7825.466 & $\min$ \\
\hline 12 & 1989 Oct. 29 & 7829.521 & $\max$ & 29 & 1989 Oct. 26 & 7826.413 & $\min$ \\
\hline 13 & 1990 Oct. 11 & 8176.547 & $\max$ & 30 & 1989 Oct. 29 & 7829.465 & $\min$ \\
\hline 14 & 1990 Oct. 13 & 8178.538 & $\max$ & 31 & 1990 Oct. 11 & 8176.470 & $\min$ \\
\hline 15 & 1990 Oct. 14 & 8178.651 & $\max$ & 32 & 1990 Oct. 13 & 8178.590 & $\min$ \\
\hline 16 & 1990 Oct. 15 & 8180.527 & $\max$ & 33 & 1990 Oct. 15 & 8180.460 & $\min$ \\
\hline 17 & 1991 Jan. 25 & 8282.262 & $\max$ & 34 & 1990 Oct. 15 & 8180.589 & $\min$ \\
\hline 18 & 1991 Jan. 26 & 8283.293 & $\max$ & 35 & 1993 Oct. 27 & 9288.440 & $\min$ \\
\hline
\end{tabular}

confidence that the cycles were counted accurately. The following ephemeris were obtained:

$M_{\min }[\mathrm{HJD}]=2447767.4869+0.132705 E$.

The (O-C) residuals with these elements are less than 0.1 which is in good agreement with the accuracy of other already published determinations. The same procedure, applied to the moments of maxima, gave best results with $P=0$ d 1333 . The $(\mathrm{O}-\mathrm{C})$ residuals of this period are also less than 0.1 . We should mention that two moments of maximal brightness (numbers 10, 12 in Table 2) occurred at the beggining or at the end of the observing runs and for the exact determination of the moments we had to perform some extrapolation of the data. This could result in a certain enhanced error in these determinations. Thus we consider the period based on the moments of minima more reliable. In a sense, the difference between the results of the analysis of moments of minimal and maximal brightness gives an estimation of the accuracy when we work with a small number of points.

The 1990/91 season is best covered by our observations. Seven observation runs were performed during the season, five of them in consecutive nights. Six moments of maximal brightness in the observing runs 18, 20, 21, 22, 23 and 24 (Table 1) were found. They are presented in Table 2 under numbers 13-18. The least squares method determination gives the elements

$$
M_{\max }[\mathrm{HJD}]=2448176.5434+0.132626 E .
$$

The $(\mathrm{O}-\mathrm{C})$ residuals calculated by these elements were less than 0.11. As for the 1989/90 season, however, the moments of extremal brightness were few. Unfortunately, we have not found any published moments of extrema for this observing season which would enable us to determine the period more accurately.

The values of the 3.2-hour photometrical period we obtained for the 1987/88, 1989/90 and 1990/91 seasons are not significantly different from the values obtained by other authors for other seasons. At first glance there is an impression that the period decreased from 0.132946 in the $1987 / 88$ season to $0 \mathrm{~d} 132626$ in $1990 / 91$. Although the $(\mathrm{O}-\mathrm{C})$ residuals for respective seasons (except for two in $1987 / 88$ ) were less than 0.11 , the small number of moments of extremum used in 1987/88 and 1990/91 was crucial. This decreases the reliability of the obtained results. We plotted the period vs. the season (Fig. 6) using values obtained by us and published by different authors (Smak \& Stepien 1975; Udalski 1988; Semeniuk et al. 1987; Volpi et al. 1988). It is seen that there was no tendency toward an increase or decrease of the period. Despite the large number of well-distributed moments of maximum determined in the 1985 and $1987 / 88$ seasons, the periods obtained were different 0.132765 and 0.132946 , respectively.

We tried to associate the moments of maxima published by Wenzel et al. (1986) and Udalski (1988) with the moments obtained by us. The calculated $(\mathrm{O}-\mathrm{C})$ values in the 1961-1994 interval were minimal for the period of 0 d 1327 . The $(\mathrm{O}-\mathrm{C})$ residuals from the ephemeris:

$$
M_{\max }[\mathrm{HJD}]=2437646.687+0.132699 \mathrm{E}
$$

reached to 0.36 . The period obtained by us and the (O-C) values with additional 21 moments of maximum are close to that obtained by Wenzel et al. (1992). 


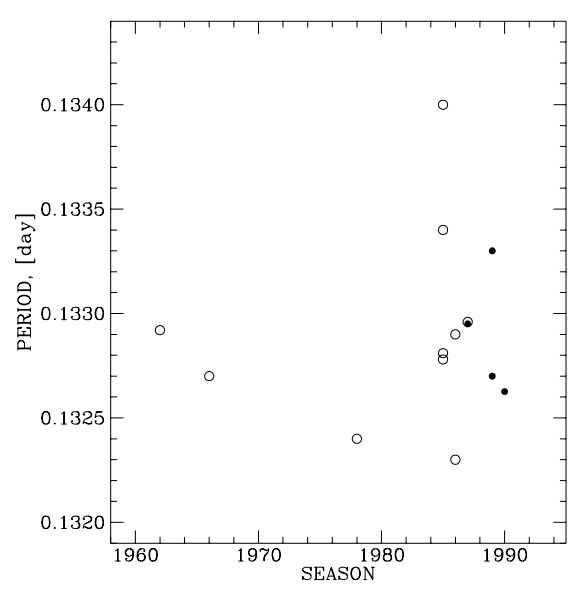

Fig. 6. The three-hour periods found for the 1961-1991 interval. - - the values obtained by us; $\circ$ - the values published by Smak \& Stepien (1975), Udalski (1988), Semeniuk et al. (1987), Volpi et al. (1988)

There are a few reasons to doubt the correctness of the period derived from the observations spanning over 33 years. Because of the short period and the rather long time interval of the investigation in which the observations occur in groups which are occasionaly separated by several years, it may have been easy to count wrong the total number of the cycles. In this case even the "goodlooking" behaviour of the (O-C) values could be caused by accidental coincidence.

\subsection{The four-day period}

We used our observing material to check the presence of a four-day brightness modulation of TT Ari. In both 1989/90 and 1990/91, we had intervals of four consecutive nights of observations that clearly showed the existence of a four-day modulation.

For a period determination, we applied the method of period dispersion minimization. Because of the large gaps in the time distribution, it was impossible to apply the maximum entropy method. For analysis the observing data sets were smoothed with a 20 min window and 5-min step. The 4-day modulation period was determined for the $1987 / 88,1989 / 90$ and $1990 / 91$ seasons because they were best covered by our observations. Observing sets 27, 11-14 and 18-22, as well as $U B V$ measurements 32 and 33 (Table 1) were used for analysis. These observations were distributed irregularly over the seasons. Thus we can expect that false frequencies might appear in our analysis and therefore we limited the interval of period search around the value of 3.76 found by Semeniuk et al. (1987) and the value determined by the equation

$\frac{1}{P_{\mathrm{b}}}=\frac{1}{P_{\mathrm{ph}}}+\frac{1}{P_{\mathrm{sp}}}$.
The time interval searched for was between 3.5 and 4.5 for all the three seasons.

The results from analysis gave periods $4.11,4.04$ and 3 d 79 for $1987 / 88,1989 / 90$ and $1990 / 91$ seasons respectively. The mean light curves based on this periods are shown in Fig. 7. The amplitudes of 4-day modulations in $U$ varied from $0 .{ }^{\mathrm{m}} 18$ in $1989 / 90$ to 0.09 in $1990 / 91$. It was 0. 14 for $1987 / 88$.

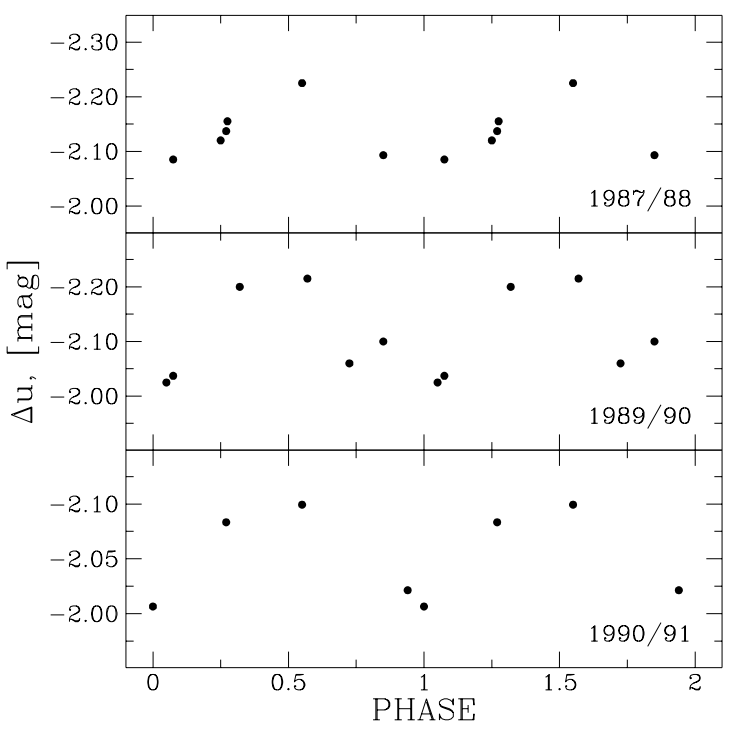

Fig. 7. The nightly average $U$ magnitudes for the $1987 / 88$, $1989 / 90,1990 / 91$ runs versus the phase of the corresponding beat period

With a mean photometric period $0 \mathrm{~d} 132699$ and an orbital period of 0.13755 Eq. (5) gave the value $P_{\mathrm{b}}=3.76$. Accounting the factors influencing the accuracy of the 3.2 hour period determination discussed in Sect. 3.3, this value is in agreement with the abovementioned values. These results confirm the existence of 4-day modulations found by Semeniuk et al. (1987). Answers to the questions associated with the long-term behaviour of the photometric periods of TT Ari can only be expected from a future intensive patrol observing programme. Performing long photoelectric series of the star in consecutive nights and a proper coverage of the observing season are highly desirable for the development of a model of this binary system.

Acknowledgements. This work was partially supported by NFSR under project No. 346/93.

\section{References}

Burg J.P., 1972, Geophys. 37, 375

Cowley A.P., Crampton D., Hutchings J.B., Marlborough J.M., 1975, ApJ 195, 413 
Götz W., 1985, Inf. Bull. Var. Stars, No. 2823

Hollander A., van Paradijs J., 1992, A\&A 265, 77

Hudec R., Huth H., Fuhrmann B., 1984, Observatory 104, 2

Kirov N.K., Antov A.P., Genkov V.V., 1991, C. R. Acad. Bulg. Sci. 44,5

Kraicheva Z., Antov A., Genkov V., 1987, Inf. Bull. Var. Stars, No. 3093

Kraicheva Z., Antov A., Genkov V., 1989, Comm. Konkoly Obs. Hung. Acad. Sci. 10, 244

Laccos R.T., 1971, Geophys. 36, 661

Ritter H., 1990, A\&AS 85, 1179

Semeniuk I., Schwarzenberg-Czerny A., Duerbeck H., et al., 1987, Acta Astron. 37, 197
Shafter A.W., Szkody P., Libert J., Penning W.R., Bond H.E., Grauer A.D., 1985, ApJ 290, 707

Smak J., Stepien K., 1969, IAU Coll. 4, 355

Stellingwerf R.F., 1978, ApJ 224, 953

Sztajno M., 1979, Inf. Bull. Var. Stars, No. 1710

Thorstensen J.R., Smak J., Hessman F.V., 1985, PASP 97, 437 Udalski A., 1988, Acta Astron. 38, 315

Volpi A., Natali G., D'Antona F., 1988, A\&A 193, 87

Wenzel W., Bojack W., Cristescu C., et al., 1986, Preprint Astron. Inst. Ondrejov, No. 38

Wenzel W., Hudec R., Schilt R., Tremko J., 1992, Contrib. Astron. Obs. Skalnate Pleso 22, 69 